PROCEEDINGS OF THE

AMERICAN MATHEMATICAL SOCIETY

Volume 133, Number 3, Pages 875-884

S 0002-9939(04)07559-8

Article electronically published on September 16, 2004

\title{
A SPECTRAL CHARACTERIZATION OF THE $H(r)$-TORUS BY THE FIRST STABILITY EIGENVALUE
}

\author{
LUIS J. ALÍAS, ABDÊNAGO BARROS, AND ALDIR BRASIL, JR. \\ (Communicated by Jon G. Wolfson) \\ Dedicated to Professor J. Lucas Barbosa on the occasion of his 60th birthday
}

\begin{abstract}
Let $M$ be a compact hypersurface with constant mean curvature immersed into the unit Euclidean sphere $\mathbb{S}^{n+1}$. In this paper we derive a sharp upper bound for the first eigenvalue of the stability operator of $M$ in terms of the mean curvature and the length of the total umbilicity tensor of the hypersurface. Moreover, we prove that this bound is achieved only for the so-called $H(r)$-tori in $\mathbb{S}^{n+1}$, with $r^{2} \leq(n-1) / n$. This extends to the case of constant mean curvature hypersurfaces previous results given by $\mathrm{Wu}$ (1993) and Perdomo (2002) for minimal hypersurfaces.
\end{abstract}

\section{INTRODUCTION}

Let $M^{n}$ be an $n$-dimensional orientable Riemannian manifold and denote by $\mathbb{S}^{n+1}$ the Euclidean sphere of constant sectional curvature one. For an isometric immersion $\psi: M^{n} \rightarrow \mathbb{S}^{n+1}$ we will denote by $A$ its second fundamental form (with respect to a globally defined normal unit vector field $N$ ) and by $H$ its corresponding mean curvature, $H=(1 / n) \operatorname{tr}(A)$. Instead of the second fundamental form, we will deal with the traceless symmetric tensor $\phi=A-H I$, the so-called traceless second fundamental form, where $I$ stands for the identity operator on $\mathcal{X}(M)$. Observe that $|\phi|^{2}=\operatorname{tr}\left(\phi^{2}\right)=|A|^{2}-n H^{2} \geq 0$, with equality if and only if $M$ is totally umbilical. For that reason, $\phi$ is also called the total umbilicity tensor of $M$. As observed by Alencar and do Carmo [1, in place of $A$, the tensor $\phi$ plays a fundamental role when extending results on minimal hypersurfaces to the case of constant mean curvature. For instance, by using the total umbilicity tensor $\phi$, Alencar and do Carmo [1] obtained the following gap theorem, which extends a well-known result on minimal hypersurfaces in the Euclidean sphere [11, 5] 8].

Received by the editors August 26, 2003 and, in revised form, October 27, 2003.

2000 Mathematics Subject Classification. Primary 53C42; Secondary 53A10.

Key words and phrases. Constant mean curvature, $H(r)$-torus, stability operator, first eigenvalue.

The first author was partially supported by DGCYT, BFM2001-2871, MCYT, and Fundación Séneca, PI-3/00854/FS/01, Spain.

The second author was partially supported by FINEP, Brazil.

The third author was partially supported by CAPES, BEX0324/02-7, Brazil. 
Theorem 1.1. Let $M^{n}$ be a compact orientable hypersurface immersed in $\mathbb{S}^{n+1}$ with constant mean curvature $H$. Assume that $|\phi|^{2} \leq B_{H}$ on $M$, where

$$
B_{H}=\frac{n}{4(n-1)}\left((n-2)|H|-\sqrt{n^{2} H^{2}+4(n-1)}\right)^{2}
$$

is the square of the positive root of the polynomial

$$
P_{H}(x)=x^{2}+\frac{n(n-2)}{\sqrt{n(n-1)}}|H| x-n\left(1+H^{2}\right) .
$$

Then

(i) either $|\phi|^{2} \equiv 0$ (and $M$ is totally umbilical), or

(ii) $|\phi|^{2} \equiv B_{H}$ and

(a) $n=2$ and $M$ is an $H(r)$-torus $\mathbb{S}^{1}(r) \times \mathbb{S}^{1}\left(\sqrt{1-r^{2}}\right)$;

(b) $n \geq 3$ and $M$ is an $H(r)$-torus $\mathbb{S}^{n-1}(r) \times \mathbb{S}^{1}\left(\sqrt{1-r^{2}}\right)$, with $r^{2} \leq$ $(n-1) / n$.

On the other hand, let $M$ be a compact minimal hypersurface in $\mathbb{S}^{n+1}$ and consider the Schrödinger operator $L=-\Delta-|A|^{2}$, where $\Delta$ is the Laplacian operator on $M$, and let $\lambda_{1}^{L}$ stands for its first eigenvalue. In [1] Simons proved that $\lambda_{1}^{L} \leq$ $-n$ if $M$ is not totally geodesic. More recently, Wu [12] obtained the following characterization of the Clifford torus by the first eigenvalue of the operator $L$.

Theorem 1.2. Let $M^{n}$ be a compact orientable minimal hypersurface immersed in the Euclidean sphere $\mathbb{S}^{n+1}$, and let $\lambda_{1}^{L}$ stand for the first eigenvalue of the Schrödinger operator $L=-\Delta-|A|^{2}$. Then

(i) either $\lambda_{1}^{L}=0$ (and $M$ is totally geodesic), or

(ii) $\lambda_{1}^{L} \leq-n$.

Moreover, $\lambda_{1}^{L}=-n$ if and only if $M$ is a Clifford torus $\mathbb{S}^{n-k}(\sqrt{(n-k) / n}) \times$ $\mathbb{S}^{k}(\sqrt{k / n})$ in $\mathbb{S}^{n+1}$.

Observe that the Schrödinger operator $L$ is closely related to the Jacobi or stability operator $J$ associated to the variational characterization of minimal hypersurfaces in $\mathbb{S}^{n+1}$, which is given by $J=-\Delta-|A|^{2}-n=L-n$. In particular, their spectra are related by the fact that $\lambda \in \operatorname{Spec}(J)$ if and only if $\lambda+n \in \operatorname{Spec}(L)$, and $\lambda_{1}^{J}=\lambda_{1}^{L}-n$. Therefore, a consequence of the result above is the following characterization of the Clifford torus by the first eigenvalue of the stability operator.

Theorem 1.3. Let $M^{n}$ be a compact orientable minimal hypersurface immersed in the Euclidean sphere $\mathbb{S}^{n+1}$, and let $\lambda_{1}^{J}$ stand for the first eigenvalue of its stability operator $J=-\Delta-|A|^{2}-n$. Then

(i) either $\lambda_{1}^{J}=-n$ (and $M$ is totally geodesic), or

(ii) $\lambda_{1}^{J} \leq-2 n$.

Moreover, $\lambda_{1}^{J}=-2 n$ if and only if $M$ is a Clifford torus in $\mathbb{S}^{n-k}(\sqrt{(n-k) / n}) \times$ $\mathbb{S}^{k}(\sqrt{k / n})$ in $\mathbb{S}^{n+1}$.

Theorem 1.3 has been recently obtained by Perdomo [10] using a new method which is completely different from Wu's method in Theorem 1.2 Motivated by Alencar and do Carmo's result Theorem 1.1 our objective here is to extend $\mathrm{Wu}$ and Perdomo's results to the case of constant mean curvature hypersurfaces in $\mathbb{S}^{n+1}$, characterizing the $H(r)$-torus in $\mathbb{S}^{n+1}$ by the first eigenvalue of the stability operator. 


\section{Statement of the main Results}

Let us recall that, for the case of constant mean curvature $H$, the stability operator is given by

$$
J=-\Delta-|\phi|^{2}-n\left(1+H^{2}\right) .
$$

The spectral behaviour of $J$ is then directly related to the instability of such hypersurfaces. Since $J$ is an elliptic operator, we can use the min-max characterization of $\lambda_{1}^{J}$, as

$$
\lambda_{1}^{J}=\min \left\{\frac{\int_{M} f J(f) \mathrm{d} v}{\int_{M} f^{2} \mathrm{~d} v} ; \quad f \in \mathcal{C}^{\infty}(M), f \not \equiv 0\right\} .
$$

Since the mean curvature $H$ is constant, it easily follows from (2.1) that

$$
\lambda_{1}^{J} \leq-n\left(1+H^{2}\right)-\frac{1}{V(M)} \int_{M}|\phi|^{2} \mathrm{~d} v \leq-n\left(1+H^{2}\right),
$$

where $V(M)$ and $\mathrm{d} v$ stand, respectively, for the $n$-dimensional volume and the volume element of $M$. Moreover, $\lambda_{1}^{J}=-n\left(1+H^{2}\right)$ if and only if $M$ is totally umbilical.

With respect to the second eigenvalue of $J$, El Soufi and Ilias 7, Theorem 2.1] have recently obtained a sharp upper bound for the second eigenvalue of a Schrödinger operator of the form $-\Delta+q$ for a compact submanifold $M^{n}$ of the Euclidean space, the hyperbolic space or the unit Euclidean sphere, where $q$ is any continuous potential on $M$, in terms of the total mean curvature of $M$ and the mean value of $q$. In particular, when $M$ is a constant mean curvature hypersurface in $\mathbb{S}^{n+1}$, and $q=-|\phi|^{2}-n\left(1+H^{2}\right)$, El Soufi and Ilias's estimate yields

$$
\lambda_{2}^{J} \leq-\frac{1}{V(M)} \int_{M}|\phi|^{2} \mathrm{~d} v \leq 0 .
$$

In particular, $\lambda_{2}^{J}$ is nonpositive and vanishes if and only if $M$ is totally umbilical. We also refer the reader to [6] for another interesting upper bound for the second eigenvalue of Schrödinger operators, given by the same authors, and its applications to the case of constant mean curvature or minimal hypersurfaces.

Remark 2.1. From a more geometrical point of view, in the case of constant mean curvature compact hypersurfaces, the stability operator $J$ is viewed as acting on smooth functions with mean value zero because the variations under consideration preserve the enclosed volume [3]. For that reason, one can consider two different eigenvalue problems: the usual Dirichlet problem, associated with the quadratic form $Q(f)=\int_{M} f J(f) \mathrm{d} v$, and the so-called twisted Dirichlet problem, associated with the same quadratic form $Q(f)$ on smooth functions subject to the additional condition $\int_{M} f \mathrm{~d} v=0$. As observed by Barbosa and Bérard [2], both eigenvalue problems are interesting, and they have similar and related properties. For instance, it easily follows from the min-max principle that their spectra are interwined by

$$
\lambda_{1}^{J}<\mu_{1}^{J} \leq \lambda_{2}^{J} \leq \mu_{2}^{J} \leq \cdots
$$

where $\mu_{i}^{J}$ denotes the eigenvalues of the twisted problem. Similarly, there are two different notions of stability, the strong stability, associated to the usual Dirichlet problem, and the weak stability, associated to the twisted Dirichlet problem. We refer the reader to [2] for a detailed study of the relationship between these two eigenvalue problems and their corresponding stability notions. 
Apart from the totally umbilical ones, the easiest constant mean curvature hypersurfaces in $\mathbb{S}^{n+1}$ are the so-called $H(r)$-tori. An $H(r)$-torus in $\mathbb{S}^{n+1}$ is obtained by considering the standard immersions $\mathbb{S}^{n-1}(r) \hookrightarrow \mathbb{R}^{n}$ and $\mathbb{S}^{1}\left(\sqrt{1-r^{2}}\right) \hookrightarrow \mathbb{R}^{2}$, for a given $0<r<1$, and taking the product immersion $\mathbb{S}^{n-1}(r) \times \mathbb{S}^{1}\left(\sqrt{1-r^{2}}\right) \hookrightarrow$ $\mathbb{S}^{n+1} \subset \mathbb{R}^{n+2}$ (see [1]). Its principal curvatures are given by

$$
\kappa_{1}=\cdots=\kappa_{n-1}=\frac{\sqrt{1-r^{2}}}{r}, \quad \kappa_{n}=-\frac{r}{\sqrt{1-r^{2}}},
$$

which implies that its constant mean curvature is

$$
H=H(r)=\frac{n-1-n r^{2}}{n r \sqrt{1-r^{2}}} .
$$

In particular, $H(r)=0$ if and only if $r^{2}=(n-1) / n$, which corresponds to the minimal Clifford torus. It follows from here that

$$
r^{2}=\frac{2(n-1)+n H^{2} \pm|H| \sqrt{n^{2} H^{2}+4(n-1)}}{2 n\left(1+H^{2}\right)},
$$

where we choose the sign as - or + according to $r^{2} \leq(n-1) / n$ or $r^{2} \geq(n-1) / n$, respectively. Moreover, $|\phi|^{2}$ is given by

$$
|\phi|^{2}=\frac{n-1}{n r^{2}\left(1-r^{2}\right)}=\frac{n}{4(n-1)}\left((n-2)|H| \pm \sqrt{n^{2} H^{2}+4(n-1)}\right)^{2},
$$

where we are using the same criterion for the sign. In particular, $|\phi|^{2}=|\phi|_{0}^{2}$ is also constant and $\lambda_{1}^{J}=-|\phi|_{0}^{2}-n\left(1+H^{2}\right)$. Therefore, if $r^{2} \leq(n-1) / n$ we have

$$
\lambda_{1}^{J}=-2 n\left(1+H^{2}\right)+\frac{n(n-2)}{\sqrt{n(n-1)}}|H||\phi|_{0},
$$

where

$$
|\phi|_{0}^{2}=\frac{n}{4(n-1)}\left((n-2)|H|-\sqrt{n^{2} H^{2}+4(n-1)}\right)^{2},
$$

and if $r^{2} \geq(n-1) / n$ we have

$$
\lambda_{1}^{J}=-2 n\left(1+H^{2}\right)-\frac{n(n-2)}{\sqrt{n(n-1)}}|H||\phi|_{0},
$$

where

$$
|\phi|_{0}^{2}=\frac{n}{4(n-1)}\left((n-2)|H|+\sqrt{n^{2} H^{2}+4(n-1)}\right)^{2}
$$

Motivated by the value of $\lambda_{1}^{J}$ for the $H(r)$-tori, we will prove the following extension of Theorems 1.2 and 1.3 .

Theorem 2.2. Let $M^{n}$ be a compact orientable hypersurface immersed in the Euclidean sphere $\mathbb{S}^{n+1}$ with constant mean curvature $H$, and let $\lambda_{1}^{J}$ stand for the first eigenvalue of its stability operator $J=-\Delta-|\phi|^{2}-n\left(1+H^{2}\right)$. Then

(i) either $\lambda_{1}^{J}=-n\left(1+H^{2}\right)$ (and $M$ is totally umbilic), or

(ii) $\lambda_{1}^{J} \leq-2 n\left(1+H^{2}\right)+\frac{n(n-2)}{\sqrt{n(n-1)}}|H| \max |\phi|$.

Moreover, $\lambda_{1}^{J}=-2 n\left(1+H^{2}\right)+\frac{n(n-2)}{\sqrt{n(n-1)}}|H| \max |\phi|$ if and only if

(a) $n=2$ and $M$ is an $H(r)$-torus $\mathbb{S}^{1}(r) \times \mathbb{S}^{1}\left(\sqrt{1-r^{2}}\right)$;

(b) $n \geq 3$ and $M$ is an $H(r)$-torus $\mathbb{S}^{n-1}(r) \times \mathbb{S}^{1}\left(\sqrt{1-r^{2}}\right)$, with $r^{2} \leq(n-1) / n$. 
In particular, Theorem 2.3 in [10] has the following extension to the constant mean curvature case.

Corollary 2.3. Let $M^{n}$ be a compact orientable hypersurface immersed in the Euclidean sphere $\mathbb{S}^{n+1}$ with constant mean curvature $H$. If $\lambda_{1}^{J}=-2 n\left(1+H^{2}\right)+$ $\frac{n(n-2)}{\sqrt{n(n-1)}}|H| \max |\phi|$, then

(a) $n=2$ and $M$ is an $H(r)$-torus $\mathbb{S}^{1}(r) \times \mathbb{S}^{1}\left(\sqrt{1-r^{2}}\right)$;

(b) $n \geq 3$ and $M$ is an $H(r)$-torus $\mathbb{S}^{n-1}(r) \times \mathbb{S}^{1}\left(\sqrt{1-r^{2}}\right)$, with $r^{2} \leq(n-1) / n$.

\section{Proof of Theorem 2.2}

Our Theorem 2.2 will be an application of the following result concerning the first eigenvalue of the Jacobi operator $J$.

Proposition 3.1. Let $M^{n}$ be a compact orientable hypersurface immersed in the Euclidean sphere $\mathbb{S}^{n+1}$ with constant mean curvature $H$, and assume that $M$ is not totally umbilic. If $\lambda_{1}^{J}$ stands for the first eigenvalue of its Jacobi operator $J=-\Delta-|\phi|^{2}-n\left(1+H^{2}\right)$, then

$$
\lambda_{1}^{J} \leq-2 n\left(1+H^{2}\right)+\frac{n(n-2)}{\sqrt{n(n-1)}}|H| \frac{\int_{M}|\phi|^{3} \mathrm{~d} v}{\int_{M}|\phi|^{2} \mathrm{~d} v}-\frac{2}{n+2} \frac{\int_{M}|\nabla \phi|^{2} \mathrm{~d} v}{\int_{M}|\phi|^{2} \mathrm{~d} v}
$$

where $\mathrm{d} v$ stands for the volume element of $M^{n}$.

In order to derive Proposition 3.1, we will also need the following auxiliary result.

Lemma 3.2 (44]12]). Let $M$ be a Riemannian manifold, and consider $\phi: \mathcal{X}(M) \rightarrow$ $\mathcal{X}(M)$ to be a traceless symmetric tensor on $M$ that satisfies the Codazzi equation, that is, $\nabla \phi(X, Y)=\nabla \phi(Y, X)$ for every $X, Y \in \mathcal{X}(M)$. Then

$$
\left.\left.|\nabla| \phi\right|^{2}\right|^{2} \leq \frac{4 n}{n+2}|\phi|^{2}|\nabla \phi|^{2} .
$$

Proof of Proposition 3.1. For every $\varepsilon>0$, let us consider the smooth function $f_{\varepsilon}=$ $\sqrt{\varepsilon+|\phi|^{2}}$. Since $M^{n}$ is not totally umbilic, we get $\lim _{\varepsilon \rightarrow 0} \int_{M} f_{\varepsilon}^{2} \mathrm{~d} v=\int_{M}|\phi|^{2} \mathrm{~d} v>$ 0 , and we can use $f_{\varepsilon}$ as a test function to estimate $\lambda_{1}^{J}$. Observe that

$$
\Delta f_{\varepsilon}=\frac{1}{2 \sqrt{\varepsilon+|\phi|^{2}}} \Delta|\phi|^{2}-\left.\left.\frac{1}{4\left(\varepsilon+|\phi|^{2}\right)^{3 / 2}}|\nabla| \phi\right|^{2}\right|^{2} .
$$

Now let us recall the following Simons type formula for the Laplacian of $|\phi|^{2}$, which for the case of a constant mean curvature hypersurface in $\mathbb{S}^{n+1}$ reads as follows (see, for instance, [1, taking into account the different choice of sign in their definition of $\phi)$ :

$$
\frac{1}{2} \Delta|\phi|^{2}=|\nabla \phi|^{2}+|\phi|^{2}\left(n\left(1+H^{2}\right)-|\phi|^{2}\right)+n H \operatorname{tr}\left(\phi^{3}\right) .
$$

Therefore, using (3.1) and (3.2), we obtain that

$$
f_{\varepsilon} \Delta f_{\varepsilon}=|\phi|^{2}\left(n\left(1+H^{2}\right)-|\phi|^{2}\right)+n H \operatorname{tr}\left(\phi^{3}\right)+|\nabla \phi|^{2}-\left.\left.\frac{1}{4\left(\varepsilon+|\phi|^{2}\right)}|\nabla| \phi\right|^{2}\right|^{2} .
$$


On the one hand, Lemma 3.2 yields

$$
\begin{aligned}
|\nabla \phi|^{2}-\left.\left.\frac{1}{4\left(\varepsilon+|\phi|^{2}\right)}|\nabla| \phi\right|^{2}\right|^{2} & \geq\left(1-\frac{n}{n+2} \frac{|\phi|^{2}}{\left(\varepsilon+|\phi|^{2}\right)}\right)|\nabla \phi|^{2} \\
& \geq\left(1-\frac{n}{n+2}\right)|\nabla \phi|^{2}=\frac{2}{n+2}|\nabla \phi|^{2} .
\end{aligned}
$$

This allows us to estimate the last two terms in (3.3). On the other hand, the socalled Okumura Lemma (see [9] as well as Lemma (2.6) in [1]) allows us to estimate the term with $\operatorname{tr}\left(\phi^{3}\right)$ in (3.3) as follows:

$$
n H \operatorname{tr}\left(\phi^{3}\right) \geq-n|H|\left|\operatorname{tr}\left(\phi^{3}\right)\right| \geq-\frac{n(n-2)}{\sqrt{n(n-1)}}|H||\phi|^{3} .
$$

Summing up, we obtain that

$$
f_{\varepsilon} \Delta f_{\varepsilon} \geq-|\phi|^{2}\left(|\phi|^{2}+\frac{n(n-2)}{\sqrt{n(n-1)}}|H||\phi|-n\left(1+H^{2}\right)\right)+\frac{2}{n+2}|\nabla \phi|^{2} .
$$

Thus,

$$
\begin{aligned}
f_{\varepsilon} J\left(f_{\varepsilon}\right) & =-f_{\varepsilon} \Delta f_{\varepsilon}-\left(|\phi|^{2}+n\left(1+H^{2}\right)\right) f_{\varepsilon}^{2} \\
& \leq|\phi|^{2}\left(|\phi|^{2}+\frac{n(n-2)}{\sqrt{n(n-1)}}|H||\phi|-n\left(1+H^{2}\right)\right) \\
& -\left(|\phi|^{2}+n\left(1+H^{2}\right)\right)\left(\varepsilon+|\phi|^{2}\right)-\frac{2}{n+2}|\nabla \phi|^{2} .
\end{aligned}
$$

Therefore, using (2.1) with $f_{\varepsilon}$ as a test function, we obtain that

$$
\begin{aligned}
\lambda_{1}^{J} \int_{M} f_{\varepsilon}^{2} \mathrm{~d} v & =\lambda_{1}^{J} \int_{M}\left(\varepsilon+|\phi|^{2}\right) \mathrm{d} v \leq \int_{M} f_{\varepsilon} J\left(f_{\varepsilon}\right) \mathrm{d} v \\
& \leq \int_{M}|\phi|^{2}\left(|\phi|^{2}+\frac{n(n-2)}{\sqrt{n(n-1)}}|H||\phi|-n\left(1+H^{2}\right)\right) \mathrm{d} v \\
& -\int_{M}\left(|\phi|^{2}+n\left(1+H^{2}\right)\right)\left(\varepsilon+|\phi|^{2}\right) \mathrm{d} v-\int_{M} \frac{2}{n+2}|\nabla \phi|^{2} \mathrm{~d} v .
\end{aligned}
$$

Finally, letting $\varepsilon \rightarrow 0$ in this last inequality we have

$$
\begin{aligned}
\lambda_{1}^{J} \int_{M}|\phi|^{2} \mathrm{~d} v \leq & -2 n\left(1+H^{2}\right) \int_{M}|\phi|^{2} \mathrm{~d} v+\frac{n(n-2)}{\sqrt{n(n-1)}}|H| \int_{M}|\phi|^{3} \mathrm{~d} v \\
& -\int_{M} \frac{2}{n+2}|\nabla \phi|^{2} \mathrm{~d} v
\end{aligned}
$$

which completes the proof of Proposition 3.1.

Now we are ready to prove our Theorem 2.2. In fact, if $M$ is totally umbilical in $\mathbb{S}^{n+1}$, then $|\phi|^{2} \equiv 0$ and $J=-\Delta-n\left(1+H^{2}\right)$, where $H$ is constant, so that $\lambda_{1}^{J}=\lambda_{1}^{-\Delta}-n\left(1+H^{2}\right)=-n\left(1+H^{2}\right)$, whose corresponding eigenfunctions are the constant functions. On the other hand, when $M$ is not umbilical, we easily deduce 
from Proposition 3.1 that

$$
\begin{aligned}
\lambda_{1}^{J} & \leq-2 n\left(1+H^{2}\right)+\frac{n(n-2)}{\sqrt{n(n-1)}}|H| \frac{\int_{M}|\phi|^{3} \mathrm{~d} v}{\int_{M}|\phi|^{2} \mathrm{~d} v}-\frac{2}{n+2} \frac{\int_{M}|\nabla \phi|^{2} \mathrm{~d} v}{\int_{M}|\phi|^{2} \mathrm{~d} v} \\
& \leq-2 n\left(1+H^{2}\right)+\frac{n(n-2)}{\sqrt{n(n-1)}}|H| \max |\phi|,
\end{aligned}
$$

as desired. Moreover, if $\lambda_{1}^{J}=-2 n\left(1+H^{2}\right)+\frac{n(n-2)}{\sqrt{n(n-1)}}|H| \max |\phi|$, then $|\nabla \phi| \equiv 0$, and Lemma 3.2 implies that $|\phi|=|\phi|_{0}$ is a positive constant. Therefore, $J=$ $-\Delta-\left(|\phi|_{0}^{2}+n\left(1+H^{2}\right)\right)$, where $|\phi|_{0}^{2}+n\left(1+H^{2}\right)$ is constant, and

$$
\lambda_{1}^{J}=\lambda_{1}^{-\Delta}-\left(|\phi|_{0}^{2}+n\left(1+H^{2}\right)\right)=-\left(|\phi|_{0}^{2}+n\left(1+H^{2}\right)\right) .
$$

On the other hand, we also have that

$$
\begin{aligned}
\lambda_{1}^{J} & =-2 n\left(1+H^{2}\right)+\frac{n(n-2)}{\sqrt{n(n-1)}}|H| \max |\phi| \\
& =-2 n\left(1+H^{2}\right)+\frac{n(n-2)}{\sqrt{n(n-1)}}|H||\phi|_{0} .
\end{aligned}
$$

Finally, from (3.5) and (3.6), it follows that

$$
P_{H}\left(|\phi|_{0}\right)=|\phi|_{0}^{2}+\frac{n(n-2)}{\sqrt{n(n-1)}}|H||\phi|_{0}-n\left(1+H^{2}\right)=0,
$$

that is, $|\phi|_{0}^{2}=B_{H}$, and by Theorem 1.1, we can conclude that $M$ is an $H(r)$-torus $\mathbb{S}^{n-1}(r) \times \mathbb{S}^{1}\left(\sqrt{1-r^{2}}\right)$, with $r^{2} \leq(n-1) / n$ provided $n \geq 3$.

Conversely, we already know that for every $H(r)$-torus $\mathbb{S}^{n-1}(r) \times \mathbb{S}^{1}\left(\sqrt{1-r^{2}}\right) \subset$ $\mathbb{S}^{n+1},|\phi|^{2}$ is constant and $\lambda_{1}^{J}=-|\phi|^{2}-n\left(1+H^{2}\right)$. The case where $r^{2} \leq(n-1) / n$ corresponds precisely to the case where the constant value $|\phi|$ is the positive root of $P_{H}$, and in that case we have that

$$
\begin{aligned}
\lambda_{1}^{J} & =-|\phi|^{2}-n\left(1+H^{2}\right)=-2 n\left(1+H^{2}\right)+\frac{n(n-2)}{\sqrt{n(n-1)}}|H||\phi| \\
& =-2 n\left(1+H^{2}\right)+\frac{n(n-2)}{\sqrt{n(n-1)}}|H| \max |\phi|,
\end{aligned}
$$

as desired. This finishes the proof of Theorem 2.2

\section{Another proof of Corollary 2.3}

Our proof of Theorem 2.2 given in the previous section relies on the classical minmax characterization of $\lambda_{1}^{J}$, and it has the advantage of providing us with both a sharp inequality for $\lambda_{1}^{J}$ and the characterization of the case where equality holds. In this section we would like to show how Perdomo's technique, which is based on a maximum principle, also works to characterize the equality case (Corollary 2.3). To the authors, this is interesting because, besides providing one with an alternative proof of the equality characterization, it could serve to better understand other similar problems, as well as to have a more global perspective of our result. In order to do that, we need the following extension of Lemma 2.1 in [10] to the case of constant mean curvature. 
Lemma 4.1. Let $M^{n}$ be an orientable hypersurface immersed in the Euclidean sphere $\mathbb{S}^{n+1}$ with constant mean curvature $H$, and let $\mathcal{U}$ be the set of non-umbilic points of $M, \mathcal{U}=\{p \in M:|\phi(p)|>0\}$. Then

$$
\Delta|\phi| \geq-|\phi| P_{H}(|\phi|)=-|\phi|\left(|\phi|^{2}+\frac{n(n-2)}{\sqrt{n(n-1)}}|H||\phi|-n\left(1+H^{2}\right)\right)
$$

on $\mathcal{U}$. Moreover, if equality holds at a point $p \in \mathcal{U}$, then $\nabla \phi(p)=0$.

Observe here that, in the case where equality holds at a point $p$, we are able to conclude not only that $\nabla \phi(p)=\beta \phi(p)$ for some linear function $\beta$ on $T_{p} M$ (see Lemma 2.1 in 10]), but that $\nabla \phi(p)=0$ indeed. This will be key in our proof of Corollary 2.3.

Proof. Assume that $M$ is not totally umbilical; otherwise, $\mathcal{U}=\emptyset$ and there is nothing to do. Observe that $\mathcal{U} \subset M$ is an open subset and $|\phi| \in \mathcal{C}^{\infty}(\mathcal{U})$. Therefore, writing $|\phi|=\sqrt{|\phi|^{2}}$ and using the Simons type formula given at (3.2) we obtain that

$$
\begin{aligned}
\Delta|\phi| & =\Delta \sqrt{|\phi|^{2}}=\frac{1}{2|\phi|} \Delta|\phi|^{2}-\left.\left.\frac{1}{4|\phi|^{3}}|\nabla| \phi\right|^{2}\right|^{2} \\
(4.1) & =|\phi|\left(n\left(1+H^{2}\right)-|\phi|^{2}\right)+\frac{1}{|\phi|} n H \operatorname{tr}\left(\phi^{3}\right)+\frac{1}{|\phi|}\left(|\nabla \phi|^{2}-\left.\left.\frac{1}{4|\phi|^{2}}|\nabla| \phi\right|^{2}\right|^{2}\right)
\end{aligned}
$$

on the set $\mathcal{U}$ of non-umbilic points of $M$. Let us now proceed similarly as in the proof of Proposition 3.1. By Lemma 3.2 we know that

$$
|\nabla \phi|^{2}-\left.\left.\frac{1}{4|\phi|^{2}}|\nabla| \phi\right|^{2}\right|^{2} \geq\left(1-\frac{n}{n+2}\right)|\nabla \phi|^{2}=\frac{2}{n+2}|\nabla \phi|^{2}
$$

on $\mathcal{U}$. On the other hand, by (3.4) we also have that

$$
\frac{1}{|\phi|} n H \operatorname{tr}\left(\phi^{3}\right) \geq-\frac{n(n-2)}{\sqrt{n(n-1)}}|H||\phi|^{2},
$$

so that

$$
\begin{aligned}
|\phi|(n & \left.\left(1+H^{2}\right)-|\phi|^{2}\right)+\frac{1}{|\phi|} n H \operatorname{tr}\left(\phi^{3}\right) \\
& \geq-|\phi|\left(|\phi|^{2}+\frac{n(n-2)}{\sqrt{n(n-1)}}|H||\phi|-n\left(1+H^{2}\right)\right) \\
& =-|\phi| P_{H}(|\phi|) .
\end{aligned}
$$

Therefore, using (4.2) and (4.3) into (4.1) we have

$$
\Delta|\phi| \geq \frac{2}{(n+2)|\phi|}|\nabla \phi|^{2}-|\phi| P_{H}(|\phi|) \geq-|\phi| P_{H}(|\phi|)
$$

on the set $\mathcal{U}$. Finally, observe that if equality holds at a point $p \in \mathcal{U}$, then $\nabla \phi(p)=0$ necessarily.

Now we are ready to prove Corollary 2.3 by using Perdomo's technique. Let us assume that $\lambda_{1}^{J}=-2 n\left(1+H^{2}\right)+\frac{n(n-2)}{\sqrt{n(n-1)}}|H| \max |\phi|$. In particular, $M$ is not totally umbilical. As is well known, the first eigenvalue $\lambda_{1}^{J}$ is simple and its eigenspace 
is generated by a first positive eigenfunction $\rho \in \mathcal{C}^{\infty}(M)$. Then $J \rho=\lambda_{1}^{J} \rho$ or, equivalently,

$$
\Delta \rho=-\left(\lambda_{1}^{J}+|\phi|^{2}+n\left(1+H^{2}\right)\right) \rho
$$

Observe that

$$
\Delta \rho^{-1}=-\rho^{-2} \Delta \rho+2 \rho^{-3}|\nabla \rho|^{2}=\left(\lambda_{1}^{J}+|\phi|^{2}+n\left(1+H^{2}\right)\right) \rho^{-1}+2 \rho^{-3}|\nabla \rho|^{2} .
$$

Now for the smooth function $f=|\phi| \rho^{-1}$ defined on $\mathcal{U}$, it easily follows that

$$
\nabla f=\rho^{-1} \nabla|\phi|-|\phi| \rho^{-2} \nabla \rho,
$$

and, using Lemma 4.1, a straightforward computation on $\mathcal{U}$ yields

$$
\begin{aligned}
\Delta f & =\Delta\left(|\phi| \rho^{-1}\right)=|\phi| \Delta \rho^{-1}+\rho^{-1} \Delta|\phi|+2\left\langle\nabla|\phi|, \nabla \rho^{-1}\right\rangle \\
& =|\phi|\left(\lambda_{1}^{J}+|\phi|^{2}+n\left(1+H^{2}\right)\right) \rho^{-1}+\rho^{-1} \Delta|\phi| \\
& +2|\phi| \rho^{-3}|\nabla \rho|^{2}-2 \rho^{-2}\langle\nabla|\phi|, \nabla \rho\rangle \\
& =|\phi| \rho^{-1}\left(\lambda_{1}^{J}+|\phi|^{2}+n\left(1+H^{2}\right)\right)+\rho^{-1} \Delta|\phi|-2 \rho^{-1}\langle\nabla f, \nabla \rho\rangle \\
& \geq|\phi| \rho^{-1}\left(\lambda_{1}^{J}+2 n\left(1+H^{2}\right)-\frac{n(n-2)}{\sqrt{n(n-1)}}|H||\phi|\right)-2 \rho^{-1}\langle\nabla f, \nabla \rho\rangle \\
& =f \frac{n(n-2)}{\sqrt{n(n-1)}}|H|(\max |\phi|-|\phi|)-2 \rho^{-1}\langle\nabla f, \nabla \rho\rangle \\
& \geq-2 \rho^{-1}\langle\nabla f, \nabla \rho\rangle .
\end{aligned}
$$

Summing up,

$$
\Delta f+2 \rho^{-1}\langle\nabla f, \nabla \rho\rangle \geq 0 \quad \text { on } \quad \mathcal{U} .
$$

Let $p_{0} \in \mathcal{U}$ be the point where the function $f$ attains its positive maximum on $M$, and let $\Omega \subset \mathcal{U}$ be a region around $p_{0}$ on which $f$ is greater than some positive constant. Since the maximum of $f$ in $\Omega$ is obtained in the interior of $\Omega$, by (4.6) and the maximum principle we deduce that $f$ is constant on $\Omega$. Since $M$ is connected, we conclude that $f$ is a positive constant on all $M=\mathcal{U}$. Therefore, $\nabla f=0$, $\Delta f=0$, and equality trivially holds in (4.6). That means that both inequalities in the computation of 4.5) must be equalities. Observe now that the first inequality in (4.5) becomes an equality if and only if equality holds in Lemma 4.1. Thus, equality in Lemma 4.1 holds at every point of $M$, and $\nabla \phi=0$ on $M$. It follows from here that $|\phi|=|\phi|_{0}$ is a positive constant. Finally, since $\Delta|\phi|=0=|\phi|_{0} P_{H}\left(|\phi|_{0}\right)$, we obtain that $|\phi|_{0}^{2}=B_{H}$, and by Theorem 1.1 we conclude the result.

Remark 4.2. Let $M^{2}$ be a compact orientable surface with constant mean curvature in $\mathbb{S}^{3}(n=2)$. Following Perdomo [10, Section 3], one can also compute from (4.4)

$$
\Delta \log \rho=\rho^{-1} \Delta \rho-\rho^{-2}|\nabla \rho|^{2}=-\left(\lambda_{1}^{J}+|\phi|^{2}+2\left(1+H^{2}\right)\right)-\rho^{-2}|\nabla \rho|^{2},
$$

and integrate on $M$ to find

$$
\alpha=\int_{M} \rho^{-2}|\nabla \rho|^{2} \mathrm{~d} v=-\left(\lambda_{1}^{J}+2\left(1+H^{2}\right)\right)|M|-\int_{M}|\phi|^{2} \mathrm{~d} v
$$

where $\alpha \geq 0$ defines a simple invariant that is independent of the choice of $\rho$ because $\lambda_{1}^{J}$ is simple. Equivalently,

$$
\lambda_{1}^{J}=-2\left(1+H^{2}\right)-\frac{1}{|M|}\left(\alpha+\int_{M}|\phi|^{2} \mathrm{~d} v\right) .
$$


Using now the Gauss equation, one can write $|\phi|^{2}$ in terms of the mean curvature $H$ and the Gaussian curvature $K$ of the surface as $|\phi|^{2}=2\left(1+H^{2}-K\right)$, and using now the Gauss-Bonnet theorem the formula above becomes

$$
\lambda_{1}^{J}=-4\left(1+H^{2}\right)-\frac{1}{|M|}(\alpha+8 \pi(g-1)),
$$

where $g$ denotes the genus of the surface. This extends Proposition 3.2 in [10] to the constant mean curvature case. Reasoning now as in the proof of Corollary 3.3 in [10, formula (4.7) allows one to give a new proof of Corollary 2.3] in the case where $n=2$, which is specific for the 2-dimensional case. Simply recall that $M$ being non-totally umbilical implies that $g \geq 1$, as in the minimal case.

\section{ACKNOWLEDGEMENTS}

This work was done while the third author was visiting the Departamento de Matemáticas of the Universidad de Murcia, Spain, as a postdoctoral fellow. He would like to thank that institution for its wonderful hospitality.

\section{REFERENCES}

[1] H. Alencar and M. do Carmo, Hypersurfaces with constant mean curvature in spheres, Proc. Amer. Math. Soc. 120 (1994), 1223-1229. MR94f:53108

[2] J.L. Barbosa and P. Bérard, Eigenvalue and "twisted" eigenvalue problems. Applications to CMC surfaces, J. Math. Pures Appl. (9) 79 (2000), 427-450. MR2001f:58064

[3] J.L. Barbosa, M. do Carmo and J. Eschenburg, Stability of hypersurfaces with constant mean curvature in Riemannian manifolds, Math. Z 197 (1988), 123-138. MR.88m:53109

[4] A. Barros, A. Brasil, Jr. and L.A.M. Sousa, Jr., A new characterization of submanifolds with parallel mean curvature vector in $\mathbb{S}^{n+p}$, Kodai. Math. J. 27 (2004), 45-56. MR2042790

[5] S.S. Chern, M. do Carmo, and S. Kobayashi, Minimal submanifolds of a sphere with second fundamental form of constant length. 1970 Functional Analysis and Related Fields (Proc. Conf. for M. Stone, Univ. Chicago, Chicago, Ill., 1968) Springer, New York, pp. 59-75. MR 42:8424

[6] A. El Soufi and S. Ilias, Majoration de la seconde valeur propre d'un opérateur de Schrödinger sur une variété compacte et applications, J. Funct. Anal. 103 (1992), 294-316. MR.93g:58150

[7] A. El Soufi and S. Ilias, Second eigenvalue of Schrödinger operators and mean curvature, Comm. Math. Phys. 208 (2000), 761-770. MR2001g:58050

[8] H.B. Lawson Jr., Local rigidity theorems for minimal hypersurfaces, Ann. of Math. (2) 89, 187-197. MR 38:6505

[9] M. Okumura, Hypersurfaces and a pinching problem on the second fundamental tensor, Amer. J. Math. 96 (1974), 207-213. MR.50:5701

[10] O. Perdomo, First stability eigenvalue characterization of Clifford hypersurfaces, Proc. Amer. Math. Soc. 130 (2002), 3379-3384. MR2003f:53109

[11] J. Simons, Minimal varieties in Riemannian manifolds, Ann. of Math. (2), 88 (1968), 62-105. MR.38:1617

[12] C. Wu, New characterizations of the Clifford tori and the Veronese surface, Arch. Math. (Basel), 61 (1993), 277-284. MR.94h:53084

Departamento de Matemáticas, Universidad de Murcia, Campus de Espinardo, E-30100 Espinardo, Murcia, Spain

E-mail address: 1jalias@um.es

Departamento de Matemática, Universidade Federal do Ceará, Campus do Pici, 60455-760 Fortaleza-Ce, BRAZiL

E-mail address: abbarros@mat.ufc.br

Departamento de Matemática, Universidade Federal do Ceará, Campus do Pici, 60455-760 Fortaleza-Ce, BraziL

E-mail address: aldir@mat.ufc.br 